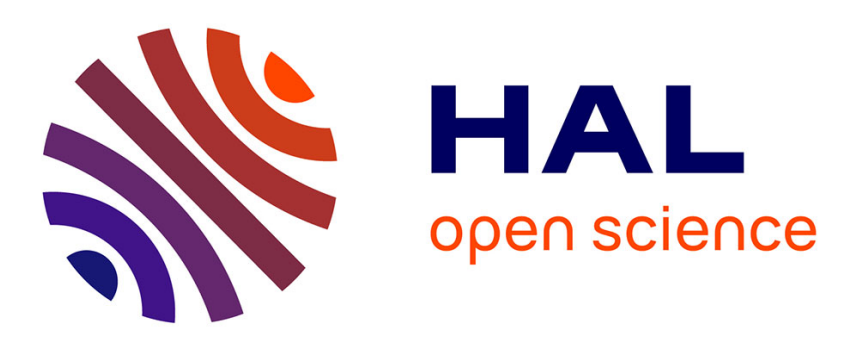

\title{
Données nouvelles sur la sécrétion phéromonale de Cryptophlebia leucotreta (Meyr.) (Lepidoptera, Tortricidae)
}

Pierre Zagatti, Bernard Lalanne-Cassou, Charles Descoins, Martine Gallois

\section{- To cite this version:}

Pierre Zagatti, Bernard Lalanne-Cassou, Charles Descoins, Martine Gallois. Données nouvelles sur la sécrétion phéromonale de Cryptophlebia leucotreta (Meyr.) (Lepidoptera, Tortricidae). Agronomie, 1983, 3 (1), pp.75-80. hal-00884487

\section{HAL Id: hal-00884487 \\ https://hal.science/hal-00884487}

Submitted on 1 Jan 1983

HAL is a multi-disciplinary open access archive for the deposit and dissemination of scientific research documents, whether they are published or not. The documents may come from teaching and research institutions in France or abroad, or from public or private research centers.
L'archive ouverte pluridisciplinaire HAL, est destinée au dépôt et à la diffusion de documents scientifiques de niveau recherche, publiés ou non, émanant des établissements d'enseignement et de recherche français ou étrangers, des laboratoires publics ou privés. 


\section{Données nouvelles sur la sécrétion phéromonale de Cryptophlebia leucotreta (Meyr.) (Lepidoptera, Tortrici- dae)}

Pierre ZAGATTI, Bernard LALANNE-CASSOU, Charles DESCOINS \& Martine GALLOIS

I.N.R.A.-C.N.R.S., Laboratoire des Médiateurs chimiques, Domaine de Brouessy, Magny-les-Hameaux, F 78470 St-Rémy-lès-Chevreuse.

\section{RÉSUMÉ}

\section{Cotonnier,}

Agrumes,

Cryptophlebia leucotreta,

Tortricidae,

Phéromone sexuelle,

Olfactomètre,

Tunnel de vol.
Dans des conditions de photopériode artificielle de jour long (16:8), les femelles de Cryptophlebia leucotreta émergent 1 heure après le début de la photophase et présentent un comportement d'appel sexuel à la $4^{\mathrm{c}}$ heure de la scotophase suivante.

Une réćvaluation de la sécrétion phéromonale de cette espèce a ćté faite par GC, GC-MS ct microchimie. Il apparaît que la femelle émet à distance une phéromone composée d'acétoxy-1 dodécène-8-E ( $E_{8} D D A$ ), d'acétoxy-1 dodécène-8- $Z\left(Z_{8} D D A\right)$ et d'acétoxy-1 dodécane $\left(\mathrm{C}_{12} \mathrm{OAc}\right)$, dans les proportions suivantes : 69 , 23 et 8 p. 100.

Eprouvé sur les mâles en olfactomètre et en tunnel de vol, le mélange formé des 3 composés identifiés provoque des réponses supéricures à celles induites par le mélange $E_{8} / Z_{8} \mathrm{DDA}$. Il s'est par ailleurs avéré que des mâles âgés de 5 jours répondaient bien mieux à la phéromone que des mâles âgés de 2 jours, bien que ceux-ci aicnt déjà atteint la maturité sexuclle. Unc hypothèse expliquant ce résultat est proposée.

\section{SUMMARY}

\section{Cotton,}

Citrus,

Cryptophlebia leucotreta,

Tortricidae,

Sex pheromone,

Olfactometer.

Wind tunnel.

\begin{abstract}
New results on the sex pheromone of the false codling moth, Cryptophlebia leucotreta Meyr. (Lepidoptera, Tortricidae).

Under laboratory conditions (light period : $16 \mathrm{~h}$, dark period : $8 \mathrm{~h}$ ), femalc Cryptophlebia leucotreta emerge $1 \mathrm{~h}$ after the beginning of the light phase and calling behaviour begins at the 4th hour of the following dark phase.

It has been shown by GC, GC-MS and microchemical analysis that the sex pheromone of this species is a blend of E-8 dodecen-1-yl acetate $\left(\mathrm{E}_{8} \mathrm{DDA}\right)$, Z-8-dodecen-1-yl acetate $\left(\mathrm{Z}_{8} \mathrm{DDA}\right)$ and dodecyl acetate $\left(\mathrm{C}_{12} \mathrm{OAc}\right)$ in the ratio $69: 23: 8$. This ratio has been determined by collecting the effluvia emitted by the females with Porapak Q as adsorbent. Other extraction techniques (crude extracts and microcncapsulation technique) gave similar data, except for $\mathrm{C}_{12} \mathrm{OAc}$ which was found in larger amounts.

Behavioural tests (olfactometer and wind tunnel) have shown that the 3-component blend is more efficient in giving a male response than the blend containing only $E_{8} / Z_{8} D D A$. Further experiments have demonstrated that the responsiveness to synthetic pheromones is better for 5-day-old males than for 2-day-old males, although the latter are sexually mature. A hypothesis explaining this result is proposed.
\end{abstract}

\section{INTRODUCTION}

Cryptophlebia leucotreta Meyr. est une tordeuse très polyphage dont la répartition est restreinte à la zone éthiopienne (ainsi qu'à Madagascar mais sans que des dégâts aient été signalés (DELATTRE, comm. pers.)). Elle s'attaque au cotonnier et au maïs en Afrique de l'Ouest (STAEUbli, 1977), mais aussi aux agrumes en Afrique du Sud (HonNiBAL, comm. pers.).

La phéromone sexuelle émise par la femelle de cette espèce fut décrite par READ et al. $(1968,1974)$ comme étant l'acétoxy-1 dodécène-7-E ( $\left.\mathrm{E}_{7} \mathrm{DDA}\right)$. Ce composé s'étant révélé d'une attractivité nulle sur le terrain, (DELATTRE, comm. pers.), PERSOONS et al. (1976, 1977) ont repris l'identification de cette phéromone et ont proposé un mélange des 2 isomères géométriques $E$ et $Z$ de l'acétoxy- 1 dodécène-8 ( $\mathrm{E}_{8} \mathrm{DDA}$ et $\left.\mathrm{Z}_{8} \mathrm{DDA}\right)$.

ANGELINI et al. $(1976,1980)$ ont montré que ce mélange d'isomères en proportions $1: 1$ était efficace pour le piégeage sexuel des mâles de $C$. leucotreta, en culture cotonnière à Bouaké (Côte d'Ivoire).

Néanmoins, dans une perspective à long terme de lutte par confusion des mâles, nous avons repris l'étude détaillée de la sécrétion phéromonale émise par la femelle de cette espèce, en y recherchant d'éventuels composés minoritaires permettant de proposer un attractif optimal. 


\section{MATÉRIEL ET MÉTHODES}

\section{A. Les insectes}

Les insectes sont élevés sur milieu artificiel (ANGELINI \& LABONNE, 1970) à partir d'une souche originaire de Côte d'Ivoire (Bouaké). Les sexes sont séparés au stade nymphal et les chrysalides sont placées en chambre climatisée $\left(27^{\circ} \mathrm{C}\right.$ et $90 \mathrm{p} .100 \mathrm{H.R}$.), sous photopériode de jour long ( $16 \mathrm{~h}$ de jour, $8 \mathrm{~h}$ de nuit).

Pour observer le comportement des femelles vierges, chaque chrysalide est placée dans une boîte transparente $\left(250 \mathrm{~cm}^{3}\right)$. On isole ainsi des séries de 16 femelles, dont on note toutes les $15 \mathrm{mn}$ les émergences et, la nuit suivante, la prise de comportement d'appel. Chaque boite est munie d'un fond de papier absorbant (qui absorbe le liquide exuvial) et d'une réserve de nourriture (tampon de coton imbibé d'eau sucrée).

\section{B. Extraction des phéromones}

\section{Extraits de glandes}

La glande à phéromone de la femelle de $C$. leucotreta est un épaississement dorsal de la membrane située entre les $8^{c}$ et $9^{\text {e }}$ segments abdominaux (ZAGATTI, 1979). Ces glandes prélevées au début de la période d'appel sur des femelles vierges âgées de $43 \mathrm{~h}\left(2^{\mathrm{c}}\right.$ nuit après l'émergence) sont mises à macérer $48 \mathrm{~h}$ à $-30^{\circ} \mathrm{C}$ dans $1 \mathrm{ml}$ d'hexane. L'extrait ainsi obtenu est ensuite filtré sur laine de verre préalablement dégraissée avant d'être concentré sous un léger courant d'argon jusqu'à un volume de $100 \mu \mathrm{l}$ environ.

\section{Microencapsulation}

Trois à cinq glandes à phéromone sont prélevées sur les femelles et serties dans des microcapsules en aluminium, à la température de l'azote liquide (DESCOINS \& GalloIs, 1979). Cette technique permet l'analyse directe par chromatographie en phase gazeuse du contenu volatil présent dans la glande, sans extraction préalable.

\section{Collecte d'effluves}

Pour mettre en évidence les effluves émis à distance par les femelles, on a adapté le système mis au point par BYRNE et al. (1975) pour les Colćoptères (fig. 1) ; 74 femelles sont placées pendant une nuit dans une enceinte close $\left(1000 \mathrm{~cm}^{3}\right)$ parcourue par un flux d'air constant de $1,5 \mathrm{l} / \mathrm{mn}$. En amont et en aval de cette enceinte sont placées 2 cartouches contenant $50 \mathrm{mg}$ d'un adsorbant spécifique, le Porapak Q, préalablement conditionné sous courant d'azote à $220^{\circ} \mathrm{C}$ pendant $24 \mathrm{~h}$. L'air est aspiré par une trompe à eau et purifié par la cartouche située en amont de l'enceinte.

Après la collecte, la cartouche d'adsorbant située en aval est rincée plusieurs fois à l'hexane et la solution obtenue est concentrée sous argon à $50 \mu \mathrm{l}$ environ.

\section{Analyses physico-chimiques}

Les méthodes d'analyse physico-chimiques utilisées sont la chromatographie en phase gazeuse (GC) et le couplage chromatographie en phase gazeuse-spectrométrie de masse (GC-MS).

Le chromatographe utilisé pour analyser les extraits hexaniques (macération de glandes et collectes d'effluves) est un GIRDEL 300 utilisant des colonnes capillaires de verre apolaires ( $40 \mathrm{~m} \mathrm{Wcot,} 0,4 \mathrm{~mm}$ i.d., SE 52) ou polaires $(50 \mathrm{~m}$ Wcot, $0,4 \mathrm{~mm}$ i.d., Carbowax $20 \mathrm{M}$ ).

Les glandes encapsulées sont analysées par un chromatographe PERKIN-ELMER 3920 équipé de l'injecteur à solides MS-41 (colonne $5 \mathrm{~m}$ inox 1/8", 10 p. $100 \mathrm{SP} 2340$ sur $\mathrm{Ch} \mathrm{W}$ ).

Enfin, l'étude par GC-MS est faite avec un chromatographe CARLO ERBA 2900 (colonne $25 \mathrm{~m}$ Wcot, $0,3 \mathrm{~mm}$ i.d. Carbowax $20 \mathrm{M}$ ), couplé à un spectromètre de masse NERMAG R-10-10.

\section{Tests comportementaux}

Les études comportementales ont été réalisées dans un olfactomètre sans choix qui est un tube de verre horizontal de $65 \mathrm{~cm}$ de long et $3,5 \mathrm{~cm}$ de diamètre, parcouru par un courant d'air de 1,51/mn (LALANNE-CASSOU, 1977), et dans un tunnel de vol dérivé de celui décrit par MiLLER \& Roelofs (1978) (2 m de long, 0,64 m de large, 0,64 m de haut ; parcouru par un courant d'air laminaire de $1 \mathrm{~m} / \mathrm{s}$, sous une intensité lumineuse d'environ 1 lux). Les mâles "naïfs" (isolés des femelles dès le stade nymphal) sont lâchés à $1,70 \mathrm{~m}$ d'une source odorante qui est un papier filtre imbibé d'attractif de synthèse. Les tests sont conduits entre la $4^{\mathrm{e}}$ et la $5^{\mathrm{c}} \mathrm{h}$ de la scotophase.

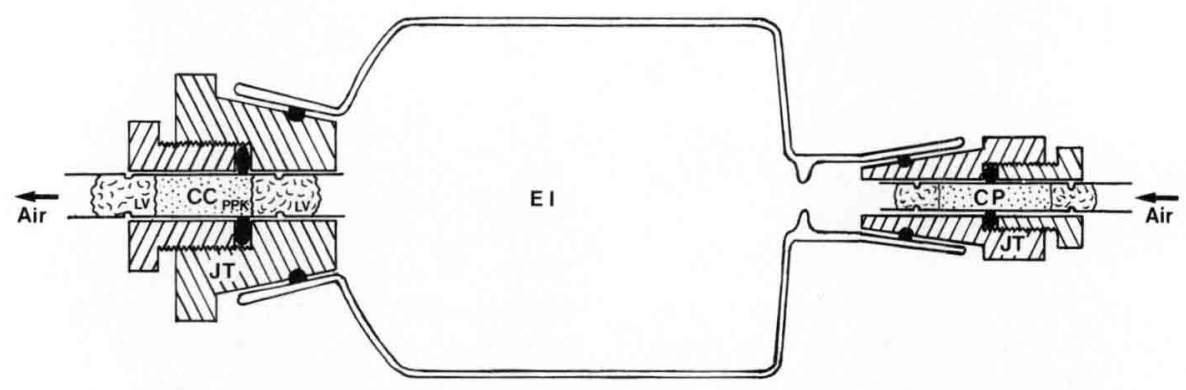

Figure 1

Collecte des effluves émis par les insectes.

$E I=$ enceinte de verre contenant les insectes;

$J T=$ joints en teflon;

$C C=$ cartouche collectrice ;

$C P=$ cartouche purificatrice

$L V=$ laine de verre :

$P P K=$ Porapak $Q$ (adsorbant).
Collection of air-borne compounds emitted by the insects $E I=$ glass jar containing the insects;

$J T=$ teflon gaskets $;$

$C C=$ collecting cartridge

$C P=$ cleansing cartridge;

$L V=$ glass wool

$P P K=$ Porapak $Q$ (adsorbent). 


\section{RÉSULTATS}

\section{A. Comportement d'appel sexuel}

La figure 2 montre, en pourcentages cumulés, les émergences des femelles adultes et la prise de comportement d'appel qui survient la nuit suivante. Une femelle en posture d'appel est reconnaissable à la position de ses ailes qui sont étendues dans un plan horizontal ou même relevées légèrement au-dessus de l'axe du corps; une extension des derniers segments abdominaux dévagine alors la glande à phéromone. On voit clairement sur la figure 2 que les émergences sont groupées à la $1^{\mathrm{rc}} \mathrm{h}$ de la photophase alors que l'appel sexuel débute à la $4^{\mathrm{c}} \mathrm{h}$ de la scotophase suivante.

L'appel de chaque femelle dure environ $90 \mathrm{mn}$, mais il n'existe aucune corrélation entre l'heure d'émergence et le début de l'appel ( $r=0,026$ (14 d.d.l.): les femelles qui émergent en premier ne sont pas forcément celles qui appellent le plus tôt).

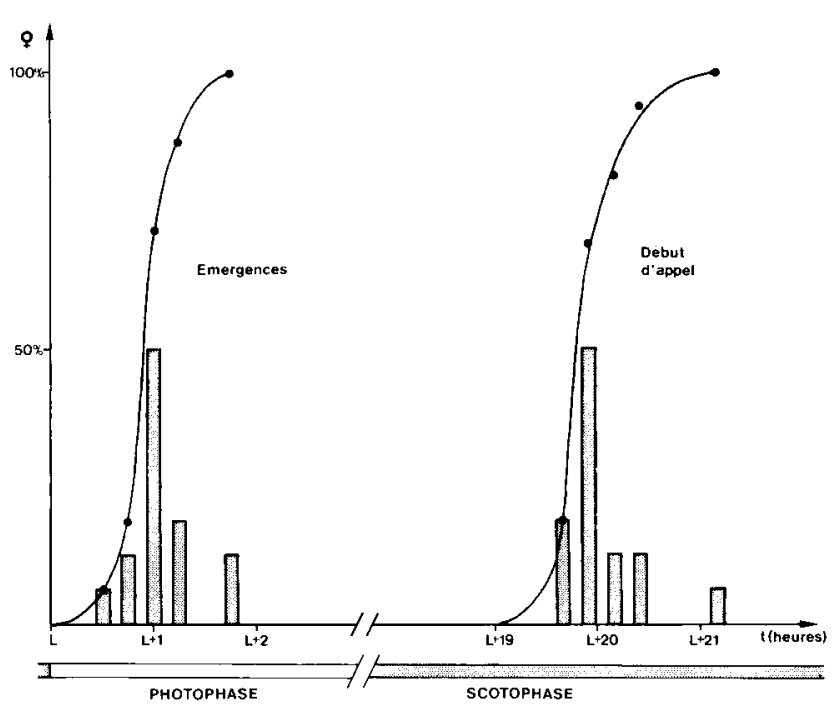

Figure 2

Emergence des femelles adultes (à gauche) et début de l'appel sexuel (à droite).

La courbe représente les fréquences cumulées

Photophase de $L \grave{a} L+16$, scotophase de $L+16 \grave{a} L+24$ (heures). Emergence of adult females (lefi) and beginning of calling behaviour (right).

The curve represents the cumulated frequencies

Light period from $L$ to $L+16$, dark period from $L+16$ to $L+24$ (hours).

\section{B. Analyse de la sécrétion des femelles}

L'analyse par GC d'un extrait obtenu par macération dans l'hexane de 330 glandes à phéromone de femelles vierges montre la présence de 3 constituants élémentaires ayant respectivement les temps de rétention de l'acétoxy-1 dodécène-8-E ( $E_{8} D D A$ : 60 p. 100), de l'acétoxy-1 dodécène-8-Z $\left(Z_{8} D D A: 23\right.$ p. 100) et de l'acétoxy-1 dodécane $\left(\mathrm{C}_{12} \mathrm{OAc}: 17\right.$ p. 100$)$, soit un rapport $\mathrm{E}_{8} / \mathrm{Z}_{8}$ DDA de $72 / 28$.

La microencapsulation de quelques glandes a permis de répéter 48 fois cette analyse et de quantifier les pourcentages relatifs des deux isomères; soit $72,8 \pm 4,8 \mathrm{p} .100$ pour le $\mathrm{E}_{8} \mathrm{DDA}$ par rapport au $\mathrm{Z}_{8}$ (intervalle de confiance à $\mathrm{P}<0,05)$, ce qui correspond tout à fait au rapport trouvé dans l'extrait précédent. L'acétate saturé se retrouve dans toutes les analyses, mais à des concentrations très variables (de 3 à 25 p. 100 du mélange des 2 isomères). On remarquera ici que $C$. leucotreta est une espèce qui produit de grandes quantités de phéromone, comparativement aux autres tordeuses étudiées jusqu'à présent (FREROT comm. pers.), puisqu'on en trouve jusqu'à $800 \mathrm{ng}$ par glande.

La collecte des effluves produits par 74 femelles durant 1 nuit a également abouti à des taux relatifs voisins : $\mathrm{E}_{8}$ DDA : 68,6 p. $100 ; \mathrm{Z}_{8} \mathrm{DDA}: 22,9$ p. 100 et $\mathrm{C}_{12} \mathrm{OAc}$ : 8,5 p. 100 (75 p. 100 de $E_{8} D D A$ par rapport au $\left.Z_{8} D D A\right)$.

Le couplage GC-MS confirme bien la présence du $\mathrm{C}_{12} \mathrm{OAc}\left(\mathrm{M}^{+} \cdot-60\right)=168$ et de 2 autres acétates monoinsaturés à 12 atomes de carbone $\left(\mathbf{M}^{+} \cdot-60\right)=166$.

Pour prouver définitivement les structures des produits présents dans la sécrétion phéromonale, on a procédé à l'ozonolyse (BEROZA \& BIERL, 1967) de la fraction correspondant aux acétates en $C_{12}$, obtenue après séparation par chromatographie micropréparative (colonne $2 \mathrm{~m}$ inox $1 / 8^{\prime \prime}$, 5 p. $100 \mathrm{SE} 52$ sur GCQ, $170^{\circ} \mathrm{C}$ ) de l'extrait. Après destruction des ozonides au tétracyanoéthylène, le produit de la réaction est analysé par GC. Le chromatogramme obtenu montre la présence de l'acétoxy-1 dodécane inchangé et de l'acétoxy-1 octanal- 8 provenant de la coupure entre les carbones 8 et 9 de la double liaison $\mathrm{Z}$ ou $\mathrm{E}$ de l'acétoxy-1 dodécène-8. Aucun autre acétoxy-aldéhyde n'est détectable après ozonolyse, ce qui laisse supposer que les $E_{8}-Z_{8} D D A$ étaient les seuls acétates monoéthyléniques présents dans la sécrétion des femelles; en particulier on ne retrouve pas trace du $\mathrm{E}_{7} \mathrm{DDA}$ primitivement identifié par READ et al. $(1968 ; 1974)$.

\section{Etude comportementale}

\section{Olfactométrie}

Nos tests ont consisté à évaluer l'effet de l'addition du $\mathrm{C}_{12} \mathrm{OAc}$ au mélange $\mathrm{E}_{8} / \mathrm{Z}_{8} \mathrm{DDA}$ sur le comportement des mâles. On a également étudié l'influence de l'âge des mâles sur les réponses. Puisque, dans nos conditions d'élevage, tous les adultes (mâles et femelles) émergent $1 \mathrm{~h}$ après le début de la photophase et que les tests sont effectués entre la $4^{\mathrm{c}}$ et la $5^{\mathrm{c}} \mathrm{h}$ de la scotophase, on a comparé entre eux des mâles âgés de $43 \mathrm{~h}$ ( $2^{\mathrm{c}}$ nuit) avec des mâles âgés de $155 \mathrm{~h}$ ( $5^{\mathrm{e}}$ nuit), en supposant également que la période de réceptivité des mâles était superposée à la période d'appel sexuel des femelles.

L'utilisation de mâles de $43 \mathrm{~h}$ plutôt que de $19 \mathrm{~h}$ ( $1^{\text {rc }}$ nuit) a permis de négliger les effets d'une maturation sexuelle insuffisante sur la perception des phéromones.

En observant le comportement des mâles en réponse à l'extrait naturel de 330 glandes à phéromone de leurs femelles (à une dose de $10^{-2}$ Femelle-Equivalent (FE)), on distingue 5 stades croissants d'excitation sexuelle.

- V: Vibration très rapide des ailes

- $\mathrm{G}$ : Extension des valves génitales

- O : Orientation vers la source d'effluves

- C: Contacts avec la source d'effluves (la boule de verre de l'applicateur).

- A : Tentatives d'accouplements homosexuels ou avec la boule de l'applicateur.

Les résultats, exprimés en pourcentages de réponses observées, sont résumés sur la figure 3 . Les pourcentages suivis de lettres différentes sont significativement différents 


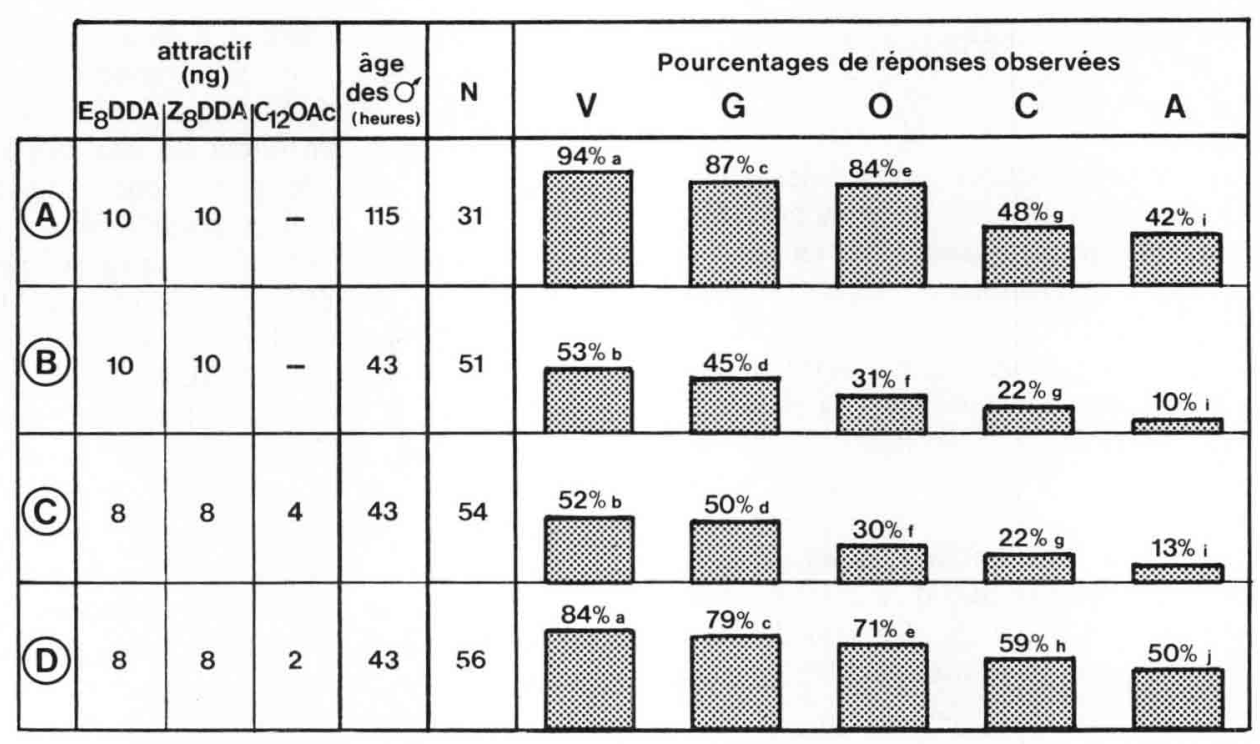

Figure 3

Réponses des mâles de $\mathrm{C}$. leucotreta observées en olfactomètre avec divers attractifs de synthèse.

$V=v i b$ ration des ailes

$G=$ extension des genitalia

$O=$ orientation vers la source

$C=$ contact avec la source

$A=$ tentative d'accouplement

Dans la même colonne, les pourcentages suivis de la même lettre ne sont pas significativement différents $(P<0,05)$.
Responses of male $\mathrm{C}$. leucotreta to synthetic attractants in olfactometer.

$V=$ wing fanning

$G=$ extension of genitalia

$O=$ orientation towards the source

$C=$ contact with the source

$A=$ copulation attempt

In the same column, percentages followed by the same letter are not significantly different $(P<0.05)$. avec $\mathrm{P}<0,05$, l'analyse statistique ayant porté sur 2 séries :

- Comparaison de A et B (U test)

- Comparaison de B, C et D (test de Friedman),

chaque étape comportementale étant analysée indépendamment des autres.

D'après la figure 3 , les réponses des mâles de $115 \mathrm{~h}$ sont largement supérieures à celles des mâles de $43 \mathrm{~h}$, et ce pour toutes les phases comportementales observées. De même, l'addition de 11 p. 100 ( $2 \mathrm{ng}$ ) de $\mathrm{C}_{12} \mathrm{OAc}$ au mélange 50/50 des $E_{8} / Z_{8} D D A(16 \mathrm{ng})$ améliore significativement les réponses, ce qui n'est pas le cas quand on en ajoute 20 p. 100 (4 ng).

\section{Tunnel de vol}

Une stimulation olfactive par l'extrait naturel de 330 glandes à phéromone $\left(2 \times 10^{-2} \mathrm{FE}\right)$ ne permet d'observer avec certitude que 2 types de réactions sexuelles chez les mâles :

- VOL : vol vers la source odorante

- ATT : atterrissage à proximité de la source.

La quasi-totalité des atterrissages observés ne se produit pas au niveau de la source même, mais $10 \mathrm{~cm}$ en dessous. Le mâle rejoint alors le papier-filtre attractif en marchant, tout en vibrant des ailes et en ouvrant ses valves génitales.

Les tests sont faits en lumière très atténuée (environ 1 lux) et les grandes dimensions du tunnel sont peu favorables à une observation détaillée des insectes, malgré l'utilisation d'un système d'enregistrement vidéo.

Trois formulations d'attractifs de synthèse ont été éprouvées sur les mâles, ce sont les mêmes que pour les tests olfactométriques, mais les doses ont été doublées (chargé totale $=40 \mathrm{ng})$.

Les résultats (tabl. 1) montrent qu'à la dose de $40 \mathrm{ng}$ il est indispensable d'utiliser un mélange phéromonal à 3 compo-

\section{TABLEAU 1}

Réponses des mâles de $\mathrm{C}$. leucotreta observées en tunnel de vol avec divers attractifs de synthèse. VOL $=$ vol soutenu, ATT $=$ atterrissage près de la source.

Responses of male $\mathrm{C}$. leucotreta to synthetic attractants in wind tunnel. $V O L=$ sustained flight, $A T T=$ landing near the source.

\begin{tabular}{cccccccc}
\hline \hline & & Attractif (ng) & $\begin{array}{c}\text { Age des } \\
\text { mâles } \\
\text { (heures) }\end{array}$ & $\mathrm{N}$ & $\begin{array}{c}\text { VOL } \\
\%\end{array}$ & $\begin{array}{c}\text { ATT } \\
\%\end{array}$ \\
& $\mathrm{E}_{8}$ DDA & Z $_{8}$ DDA & $\mathrm{C}_{12}$ OAc & & & \\
\hline $\mathrm{E}$ & 20 & 20 & - & 43 & 12 & 0 & 0 \\
$\mathrm{~F}$ & 16 & 16 & 8 & 43 & 10 & 40 & 30 \\
$\mathrm{G}$ & 16 & 16 & 4 & 43 & 20 & 45 & 25 \\
\hline
\end{tabular}

sants pour provoquer l'envol et l'atterrissage des mâles. Il n'y a pas de différence significative entre les réponses aux formulations $F$ et $G$.

Avec l'attractif $\mathrm{E}$ (mélange $\mathrm{E}_{8} / \mathrm{Z}_{8} \mathrm{DDA}$ seul), les mâles présentent des vibrations d'ailes et des extensions des valves génitales, en s'agglutinant au bord de la plate-forme de départ, vers la source d'effluves, mais aucun ne s'envole pour rejoindre cette source. Ces mâles présentent donc, sur leur plate-forme de départ, des réponses comparables à celles qu'on a pu observer dans le tube d'olfactomètre, où le vol réel ne peut être mis en évidence.

\section{DISCUSSION}

Les différentes techniques d'analyses physico-chimiques mises en œuvre confirmıent bien la présence d'un mélange phéromonal à 3 composés avec des pourcentages respectifs d'environ 65,25 et 10 p. 100 pour les $E_{8} D D A, Z_{8} D D A$ et 
$\mathrm{C}_{12} \mathrm{OAc}$, ce dernier composé apparaissant en proportions assez variables suivant les analyses. La collecte d'effluves étant la seule méthode permettant de mesurer ce que la femelle émet, on admettra que le pourcentage de $\mathrm{C}_{12} \mathrm{OAc}$ intervenant dans le message chimique est inférieur à 10 p. 100.

Les tests olfactométriques ont montré l'influence de l'âge des mâles sur les réponses sexuelles (fig. 3), les mâles de $5 \mathrm{j}$ répondent bien mieux que les mâles de $2 \mathrm{j}$ (qui sont pourtant mûrs sexuellement). Pour expliquer ce phénomène, on peut avancer l'hypothèse suivante: les mâles n'ont jamais été soumis à des effluves femelles avant le test ; c'est là une situation tout à fait improbable dans la nature, où les insectes doivent être environnés de phéromone dès la nuit qui suit leur émergence (la protandrie est peu marquée chez cette espèce). On peut donc penser que dans nos conditions d'expérience, les seuils de réceptivité doivent être beaucoup plus bas que dans la nature, et d'autant plus bas que l'on conserve longtemps les mâles isolés des effluves phéromonaux des femelles. Les réponses remarquables des mâles de $115 \mathrm{~h}$ ne seraient ainsi que le résultat d'une rétention anormale du comportement sexuel sous dépendance de la perception des phéromones. Pour la reproductibilité des tests comportementaux, il est donc indispensable que tous les sujets aient le même âge.

En olfactomètre, l'addition de 10 p. 100 de $\mathrm{C}_{12} \mathrm{OAc}$ au mélange des 2 isomères $\mathrm{E}_{8} / \mathrm{Z}_{8} \mathrm{DDA}$ augmente très sensiblement les réponses des mâles (fig. 3). Cette amélioration ne privilégie pas une phase comportementale particulière par rapport aux autres (contacts au niveau de la source, par exemple). Toutes les réponses augmentent en effet dans la même proportion quand on ajoute ce composé. On dira donc que le $\mathrm{C}_{12} \mathrm{OAc}$ agit en synergie avec les isomères $\mathrm{E}_{8}$ et $\mathrm{Z}_{8} \mathrm{DDA}$ en abaissant le seuil de réceptivité du mâle à la phéromone de la femelle.

Quand on étudie les résultats obtenus avec le tunnel de vol, on s'aperçoit que le mélange $\mathrm{E}_{8} / \mathrm{Z}_{8} \mathrm{DDA}$ seul (40 ng) ne déclenche aucun vol des mâles et qu'il suffit d'ajouter $4 \mathrm{ng}$ de $\mathrm{C}_{12} \mathrm{OAc}$ pour que l'attractif soit efficace. Or, sur le terrain, un mélange $\mathrm{E}_{8} / \mathrm{Z}_{8} \mathrm{DDA} 50-50$ sans $\mathrm{C}_{12} \mathrm{OAc}$ se révèle très attractif pour les mâles si les capsules sont fortement chargées ( 10 à $20 \mathrm{mg}$ pour des capsules polyéthylène) (ANGELINI et al., 1976, 1980). On peut donc penser qu'à de telles concentrations, ce mélange est capable d'induire chez les mâles des vols et des atterrissages, faute de quoi le piégeage serait inefficace. En tunnel de vol, il aurait sans doute suffi d'augmenter la dose de l'attractif $E$ $\left(\mathrm{E}_{8} / \mathrm{Z}_{8} \mathrm{DDA}:\right.$ : 50/50) pour faire s'envoler les mâles, là aussi nous parlerons de synergie pour qualifier le rôle du $\mathrm{C}_{12} \mathrm{OAc}$. On met ici en évidence la qualité essentielle du tunnel de vol, qui est de faire réellement voler les insectes; celui-ci apparaît donc comme un excellent moyen pour juger de l'efficacité d'un attractif avant de le proposer aux utilisateurs sur le terrain.

Cette nouvelle formulation a d'ailleurs été essayée avec succès pour le piégeage sexuel de $C$. leucotreta en culture cotonnière à Bouaké (Côte d'Ivoire) par ANGELINI et al. (1981). Il apparaît que l'addition de 10 p. 100 de $\mathrm{C}_{12} \mathrm{OAc}$ améliore significativement l'attractivité du mélange $\mathrm{E}_{8} / \mathrm{Z}_{8} \mathrm{DDA}$ pour des doses d'attractif de $2 \mathrm{mg}$ par capsule (caoutchouc).

\section{CONCLUSION}

Tout en aboutissant à préciser les composants d'un attractif sexuel naturel et à donner une formule de synthèse plus efficace sur le terrain, cette réinvestigation de la phéromone de Cryptophlebia leucotreta a permis de préciser les avantages et les limites des diverses méthodes d'étude des comportements sexuels en laboratoire. La variabilité du matériel biologique impose une grande rigueur dans la répétition des tests comportementaux pour que les résultats soient interprétables (âge des insectes, heure des tests, nécessité d'employer des doses d'attractif proches des seuils de perception). Il reste que $C$. leucotreta présente un grand nombre d'avantages - production importante de phéromone, seuils de réceptivité très bas, séquences comportementales aisées à reproduire en laboratoire, élevage facile sur milieu artificiel - qui en font un modèle remarquable pour des travaux plus fondamentaux sur la perception et les modes d'action des phéromones.

Reçu le 3 février 1982. Accepté le 14 septembre 1982.

\section{REMERCIEMENTS}

Nous tenons à remercier tout particulièrement M. R. COUILLOUD (GERDAT, Montpellier) qui nous a fourni les insectes nécessaires à cette étude, M. R. CAusse (I.N.R.A., Montfavet) pour l'expérimentation en tunnel de vol, ainsi que la Société RhônePoulenc pour l'utilisation du spectromètre de masse.

\section{RÉFÉRENCES BIBLIOGRAPHIQUES}

Angelini A., Couilloud R., Delabarre M., Lhoste J., 1976. Effet attractif des isomères de l'acétate de 8 dodécényle pour les mâles de Cryptophlebia leucotreta (Meyr.), C.R. Acad. Agric. Fr., 62 (6), 441-444.

Angelini A., Descoins C., Le Rumeur C., Lhoste J., 1980. Nouveaux résultats obtenus avec un attractif sexuel de Cryptophlebia leucotreta (Meyr.). Coton Fibres trop., 35, 277-281.

Angelini A., Descoins C., Lhoste J., Trijau J. P., Zagatti P., 1981. Etude de nouvelles formulations d'attractifs de synthèse pour le piégeage sexuel de Cryptophlebia leucotreta (Meyr.). Coton Fibres trop., 36, 259-264.

Angelini A., Labonne V., 1970. Mise au point sur l'étude de Cryptophlebia (Argyroploce) leucotreta (Meyr.) en Côte d'Ivoire. Coton Fibres trop., 25, 497-500.
Beroza M., Bierl A. B., 1967. Rapid determination of olefin position in organic compounds in microgram range by ozonolysis and gas chromatography. Anal. Chem., 39 (10), 1131-1135.

Byrne K. J., Gore W. E., Pearce G. T., Silverstein R. M., 1975. Porapak Q collection of airborne organic compounds serving as models for insect pheromones. J. Chem. Ecol., 1 (1), 1-7.

Descoins C., Gallois M., 1979. Analyse directe pár chromatographie en phase gazeuse des constituants volatils présents dans les glandes à phéromones des femelles de Lépidoptères. Ann. Zool. Ecol. anim., 11 (4), 521-532.

Lalanne-Cassou B., 1977. Contribution à l'étude de la communication sexuelle par phéromone chez l'eudémis de la vigne: Lobesia botrana Schiff. (Lepidoptère, Tortricidae, Olethreutinae). Thèse de $3^{\mathrm{c}}$ cycle, biologie animale (entomologie) Université Paris VI. 
Miller J. R., Roelofs W. L., 1978. Sustained-flight tunnel for mcasuring insect responses to wind-borne sex pheromones. $J$. Chem. Ecol., 4 (2), 187-198.

Persoons C. J., Ritter F. J., Hainaut D., Demoute J. P., 1976. Sex pheromone of the false codling moth Cryptophlebia (Argyroploce) leucotreta (Lep. Tortricidae), trans-8-dodecenyl acetate, a corrected structure. Med. Fac. Landbouww. Rijksuniv. Gent, 41 (2), 937-943.

Persoons C. J., Ritter F. J., Nooyen W. J., 1977. Sex pheromone of the false codling moth Cryptophlebia leucotreta (Lep. Tortricidae), evidence for a two-component system. J. Chem. Ecol., 3 (6), 717-722.

Read J. S., Hewitt P. H., Warren F. L., Myberg A. C., 1974. Isolation of the sex pheromone of the moth Argyroploce leucotreta. J. Insect Physiol., 20 (3), 441-450.
Read J. S., Warren F. L., Hewitt P. H., 1968. Identification of the sex pheromone of the false codling moth Argyroploce leucotreta. Chem. commun. 1968, 792-793.

Staeubli A., 1977. Contribution à l'ćtude de Cryptophlebia leucotreta (Meyr.), particulièrement au Bénin. Coton Fibres trop., 32, 325-349.

Zagatti P., 1979. Etude de la communication phéromonale chez Cryptophlebia leucotreta Meyr. (Lepidoptera, Tortricidae) et chez l'écaille fileuse, Hyphantria cunea Drury (Lepidoptera, Arctiidae) défoliateur forestier nouvellement introduit en France. Mém. DEA Entomol., Univ. Paris VI, $55 \mathrm{p}$. 